\title{
Frequency distributions: from the sun to the earth
}

\author{
N. B. Crosby \\ Belgian Institute for Space Aeronomy, Avenue Cirulaire 3, 1180 Brussels, Belgium
}

Received: 14 February 2011 - Revised: 20 June 2011 - Accepted: 20 June 2011 - Published: 4 November 2011

\begin{abstract}
The space environment is forever changing on all spatial and temporal scales. Energy releases are observed in numerous dynamic phenomena (e.g. solar flares, coronal mass ejections, solar energetic particle events) where measurements provide signatures of the dynamics. Parameters (e.g. peak count rate, total energy released, etc.) describing these phenomena are found to have frequency size distributions that follow power-law behavior. Natural phenomena on Earth, such as earthquakes and landslides, display similar power-law behavior. This suggests an underlying universality in nature and poses the question of whether the distribution of energy is the same for all these phenomena. Frequency distributions provide constraints for models that aim to simulate the physics and statistics observed in the individual phenomenon. The concept of self-organized criticality (SOC), also known as the "avalanche concept", was introduced by Bak et al. $(1987,1988)$, to characterize the behavior of dissipative systems that contain a large number of elements interacting over a short range. The systems evolve to a critical state in which a minor event starts a chain reaction that can affect any number of elements in the system. It is found that frequency distributions of the output parameters from the chain reaction taken over a period of time can be represented by power-laws. During the last decades SOC has been debated from all angles. New SOC models, as well as non-SOC models have been proposed to explain the powerlaw behavior that is observed. Furthermore, since Bak's pioneering work in 1987, people have searched for signatures of SOC everywhere. This paper will review how SOC behavior has become one way of interpreting the power-law behavior observed in natural occurring phenomenon in the Sun down to the Earth.
\end{abstract}

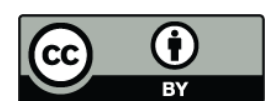

Correspondence to: N. B. Crosby (norma.crosby@oma.be)

\section{Introduction}

It is well-known that natural catastrophes occurring on Earth such as earthquakes, landslides, etc. cost many human lives each year (see for example O'Neill, 2005). Consequently, mitigation (e.g. designing earthquake resistant structures) and prediction of these hazards (e.g. earthquake - tsunami prediction) is of vital importance, especially the understanding of what triggers the extreme events. The space environment also has hazards of its own which in some circumstances may have unexpected and unwanted effects on technology and humans both in space and on Earth. In space, radiation protection from phenomena such as solar energetic particle events is a prime issue for satellites in Earth orbit and space station operations, for extended missions to planets in our solar system (e.g. Mars), or for a return visit to the Moon. Indeed the solar-terrestrial environment is truly dynamic on all temporal and spatial scales. In the rest of this text hazards shall be referred to as "avalanches".

Earthquakes are triggered when a mechanical instability occurs and a fracture (the sudden slip of a fault) appears in a part of the Earth's crust and the main question to answer is when does such an event "avalanche" happen? Geller et al. (1997) argue that it is not possible to answer this question "For large earthquakes to be predictable, they would have to be unusual events resulting from specific physical states. However, there is also a general consensus that the Earth is in a state of self-organized criticality where any small earthquake has some probability of cascading into a large event". Regarding any possible existing earthquake precursors Geller et al. (1997) also state "Thousands of observations of allegedly anomalous phenomena (seismological, geodetic, hydrological, geochemical, electromagnetic, animal behavior, and so forth) have been claimed as earthquake precursors, but in general, the phenomena were claimed as precursors after the earthquakes occurred". Furthermore, Sornette (2002) wrote "There is a series of surprizing and

Published by Copernicus Publications on behalf of the European Geosciences Union and the American Geophysical Union. 
somewhat controversial studies showing that many large earthquakes have been preceded by an increase in the number of intermediate sized events". Contrary to Sornette's statement that many large earthquakes have intermediate-sized precursors, there are new studies of earthquakes in Turkey that find a 6-yr quiescence period before large earthquakes (Oztürk, 2011).

What is the most fruitful way to study avalanches, especially the catastrophic avalanches, better known as the extreme events? Basically, there are two approaches that are used for this purpose: "case by case" or "applying statistics". Does one approach teach us more than the other or do the approaches complement each other, for example in regard to prediction purposes? Individual case studies have often been the traditional way to study extreme events. This has often been the preferred approach as they cause the most damage and there simply is more information available (data from many different sources has been recorded). For example, physical processes leading to magnetic energy releases have been analyzed in individual solar flares using multiwavelength observations. Such single-case solar flare studies constrain the number and energy spectrum of accelerated electrons and ions and the characteristics of magnetic structures at different scales in which energetic particles are produced, propagate and radiate. However single case-by-case studies can sometimes create general beliefs such as large solar flares are different from average flares, the "Big Flare Syndrome (BFS)" as termed by Kahler (1982). Though the observations may suggest this, the situation may simply be caused by not being able to measure all the associated signatures in the smaller events due to detection threshold limits. A study performed by Cliver et al. (1994) indicates that gamma-ray line (GRL) flares are not fundamentally different from other large flares without detectable GRL emission. Instead the fact that small flares lack gamma-ray observations may simply be a detection threshold effect and as was originally suggested by Forrest (1983) all flares do accelerate ions. It is now generally accepted that bigger flares are not fundamentally different from smaller flares, because the energy-related parameters follow the same (power-law) scaling.

With more and more data becoming available (huge databases covering long time spans) it is not always possible to study each "single avalanche event" and a statistical approach can be used instead. Such an approach is valid if the phenomenon being studied is found to result from the same mechanisms of energy release and in the case of solar flares is also independent on whatever their temporal and/ or spectral characteristics may be. The goal of such a statistical approach is to describe the global behavior of the avalanches by for example performing frequency distributions of parameters describing the size of the events constituting the database. A frequency distribution is a function that describes the occurrence rate of events as a function of their size, usually plotted as a histogram of the logarithmic number versus the logarithmic size. For numerous natural phenomena (e.g. earthquakes, solar flares, etc.) it is found that frequency distributions of the output parameters describing the phenomenon from the chain reaction taken over a period of time can be represented by power-laws of the form $d N=A x^{-\alpha} d x$, where $d N$ is the number of events recorded with the parameter $\mathrm{x}$ of interest between $x$ and $x+d x$, and $A$ and $\alpha$ are constants. Differential distributions are preferable as all bins of the histograms are independent of each other, whereas integral (cumulative) distributions are best for studies with poor statistics. The underlying physics determining the exact value of the spectral index of the power-law, which varies for different size parameters and phenomena, is not yet well understood, but may suggest some form of universality.

In regard to risk hazard assessment, power-law behavior also suggests that extreme avalanches are more frequent than one might have originally thought. For mitigation and prediction issues the important question is whether or not extreme events are "outliers" from the rest of the distribution. If they are not, then the physical processes governing the extreme events should be the same as those governing the average events. Therefore a frequency distribution is a powerful tool providing information about the global properties of a phenomenon over a given observational period.

Various concepts (models) have been proposed that produce power-law behavior in nature and one of these is the concept of self-organized criticality (SOC). SOC, also known as the "avalanche concept", was introduced by Bak et al. $(1987,1988)$ to characterize the behavior of dissipative systems that contain a large number of elements interacting over a short range. The systems evolve to a critical state in which a minor event starts a chain reaction that can affect any number of elements in the system. Hence, a power-law distribution results from nonlinear or coherent processes, has no characteristic spatial scale and is the hallmark of nonlinear dissipative systems. Such systems are constantly driven by some random energy input evolving into a critical state that is maintained as a power-law distribution.

In this paper frequency distributions based on observations of various avalanche phenomena that occur in the Sun-Earth scenario are presented. References to theoretical works are also included in the paper and an introduction to SOC is given in Sect. 2. In Sect. 3 various frequency distribution studies performed on phenomena occurring on the Sun (solar flares and solar energetic particle events) are shown. Section 4 discusses results obtained on solar wind and magnetospheric phenomena. Thereafter Sect. 5 presents avalanches (e.g. earthquakes) on Earth. Throughout Sects. 3, 4 and 5 the results are discussed with respect to the avalanche concept. Thereafter Sect. 6 presents how the results can be used as input for forecasting and engineering tools. Section 7 discusses the findings presented in this review paper in respect to SOC. The paper ends by listing the main conclusions found in this review study. 


\section{Self-Organized Criticality}

The concept of SOC evolved from numerical simulations utilizing several relatively simple cellular-automata models. The term cellular refers to the fact that the model is discrete concerning space and the term automaton means that the evolution of the system takes place in discrete steps which are not necessarily linked to time in the physical sense. In summary, a cellular automaton is a system which is discrete concerning space and time, and whose evolution through time is defined by some mathematical redistribution rule. SOC behavior is characterized by power-law behavior and has been observed in a large number of natural phenomena (e.g. earthquakes, solar flares, etc.). Several books have been written on SOC regarding phenomena that show this behavior as well as models that simulate SOC. For more information on SOC see the books written by Bak (1996), Hergarten (2002), Jensen (1998) and most recently the book by Aschwanden (2011) that covers SOC in astrophysics.

In 1987, Per Bak and co-workers presented a model that evolves towards a critical state without any external tuning. This model is often called Per Bak's sandpile model or the Bak-Tang-Wiesenfeld (BTW) model. In their first paper (Bak et al., 1987), the BTW model was derived from a model for the dynamics of an array of coupled pendulums. Thereafter the same model was interpreted in terms of sandpile dynamics (Bak et al., 1988). In this quadratic two-dimensional lattice model there is a square grid of boxes and at each time step a particle is dropped into a randomly selected box. When a box accumulates four particles, they are redistributed to the four adjacent boxes. If the adjacent box is an edge box the particle is lost from the grid. Redistributions can lead to further instabilities, with avalanches of particles lost from the edge of the grid. The noncumulative frequency-area distribution of model avalanches is found to satisfy a power-law (fractal) distribution. The avalanche concept can be illustrated by a simple sandpile experiment (Held et al., 1990; Bak and Chen, 1991), where sand grains are added to a sandpile until the slope of the sandpile reaches a critical value and an avalanche occurs. A natural landslide on Earth is a natural phenomenon associated with the sandpile model.

Another model that exhibits SOC is the forest-fire model (Drossel and Schwabl, 1992a, b). In the simplest version of this model, a square grid of sites is considered. At each time step either a tree is planted on a randomly chosen unoccupied site or a spark is dropped on the site. If the spark is dropped on a tree, that tree and all adjacent trees are burned in a model forest fire. It is found that the frequency area distribution of the smaller fires can be represented by a power-law. Forest fires occurring on Earth are associated with this model.

The slider-block model also exhibits SOC and in this model an array of slider blocks are connected to a constant velocity driver plate by driver springs and to each other by connector springs. The blocks exhibit stick-slip behavior due to frictional interactions with the plate across which they are pulled. The area is defined to be the number of blocks that participate in a slip event. It is found that the frequency area distribution of the smaller slip events is represented by a power-law (e.g. Carlson and Langer, 1989, Carlson et al., 1994). Earthquakes are associated with the slider-block model.

It should be mentioned that there exist other theoretical models that produce power-law behavior, for example the inverse cascade model. Small clusters of (e.g. trees) on a grid coalesce to form larger clusters, and clusters are lost in fires that occur randomly. The result is a self-similar inversecascade that satisfies an inverse power-law distribution of clusters sizes. Turcotte and Malamad (2004) have related the inverse-cascade model to the results of several cellularautomata models and also to real data observed for different natural hazards. Rosner and Vaiana (1978) developed the stochastic relaxation model to describe solar flares. In their model flaring is a stochastic process, energy build-up is exponential between flares, and all the energy built up between flares is released by the following flare whereafter the system returns to its unperturbed ground state via the flare. However, their prediction that the duration of energy storage is correlated with flare size was not confirmed by observations $(\mathrm{Lu}$, 1995; Crosby, 1996; Crosby et al., 1998; Wheatland, 2000; Georgoulis et al., 2001).

Forced SOC (FSOC), an alternative concept, shares all the avalanche phenomenology of power-law distributions, but is not necessarily self-organized (Chang, 1992, 1999). The key aspect of this FSOC model is that some external dynamics exerts forces on a system to produce power-law like distributions of avalanches without internal self-organization. Magnetic substorms seem to require a continuous loading process in order to drive them into a critical/near-critical state (Horton and Doxas, 1996). Klimas et al. (2004) performed a study of SOC in models of the magnetic field reversal and driven reconnection of the plasma sheet by performing numerical simulations with 2-D resistive MHD models that involve anomalous resistivity of a current-driven kinetic instability. They show that the Poynting flux in cascades that develop occurs in bursts, whose duration, integrated size and total energy content exhibit scale-free power-law probability distributions over large ranges of scales providing strong evidence that their model has developed into SOC.

Turbulence produces cascading of spatial scales with a spatial correlation (big eddies fragment locally into smaller eddies), while SOC avalanches are uncorrelated. Using statistical methods common to studies of SOC and intermittent turbulence (IT), Uritsky et al. (2007) analyzed extreme ultraviolet images of the solar corona. The data exhibited simultaneous characteristics of both regimes (power-law avalanche statistics as well as multiscaling of structure functions for spatial activity). This implies that both SOC and IT may be manifestations of a single complex dynamical process 
entangling avalanches of magnetic energy dissipation with turbulent particle flows.

Following Bak's above mentioned pioneering work in 1987, not only was there an "avalanche" in SOC studies from the modeling side, but people started to search for signatures of SOC everywhere in natural occuring phenomenon from the Sun to the Earth (see Sects. 3, 4 and 5).

\section{Solar phenomena}

Like on Earth, the space environment also has a weather of its own, its own space weather. Our Sun is definitely the driver of our local space weather and it is well known that the solar corona is a very dynamic region which is the source of many phenomena (e.g. solar flares, coronal mass ejections, solar energetic particle events, etc.). Therefore, it was not surprizing that it was on the Sun where one first began to search for SOC signatures. Observations have provided convincing evidence indicating certain space plasma processes are in states of complexity and SOC, especially with the discovery of the apparent power-law probability distribution of solar flare intensities.Recently Robbrecht et al. (2009) presented results that suggest the possibility that the size of coronal mass outflows also follow a power-law distribution (no typical coronal mass ejection size exists).

Overview tables of numerous distributions of peak fluxes in solar flare phenomena can be found in (Hudson, 1978; Crosby et al., 1993; Aschwanden, Dennis and Benz 1998; Charbonneau et al., 2001; Miroshnichenko et al., 2001; Aschwanden, 2004, 2011). Examples of frequency distribution studies performed on solar flares and solar energetic particle events are given in the next two sub-sections.

\subsection{Solar flares}

Solar flares are related to magnetic energy releases in a large range of sizes and occur on time scales ranging from a few seconds or less to hours. The statistical behavior of solar flares has been characterized with frequency distributions of hard X-ray parameters and here some of the pioneering references are listed: Datlowe et al., 1974; Lin et al., 1984; Dennis, 1985; Schwartz et al., 1992; Crosby et al., 1993; Lee et al., 1993; Pearce et al., 1993; Bai, 1993; Biesecker, 1994; Biesecker et al., 1994; Bromund et al., 1995; Kucera et al., 1997. It is found that most of the distributions can be represented by power-laws having a slope in the range of $-1.4, \ldots,-2.4$ above a threshold (usually attributed to the sensitivity of the experiment used).

Lu and Hamilton (1991) proposed a model based on SOC, where each solar flare is considered an avalanche event in a critically stable system. They assume that the solar coronal magnetic field is in a self-organized state where the random twisting of the magnetic field by photospheric convection motion plays the role of the addition of sand grains. The local instability is related to the magnetic discontinuity angle between the magnetic field vectors on opposite sides of a particular current sheet (Parker, 1988). When the critical angle is exceeded reconnection can proceed explosively and the energy will be "redistributed" along the magnetic field thus reducing the angle. The way the magnetic energy is redistributed (e.g. isotropically, anisotropically), how the system is driven (the loading mechanism) and the "incorporation" of magnetohydrodynamics has been further developed by authors such as Lu et al. (1993), Galsgaard (1996), Georgoulis and Vlahos (1998), Isliker et al. (2001).

Traditionally the total database of the parameter describing the solar flare is used in the analysis providing one with the global signature of the phenomenon being studied. However, Crosby et al. (1998) found that by sub-grouping a parameter as a function of another parameter describing the solar flare and thereafter performing frequency distributions on individual sub-groups provides interesting information. In Fig. 1b the frequency distribution of the deka-keV WATCH solar flare peak count rate for the total database (1990-mid1992) is illustrated. It can be represented, above the turn-over at $50 \mathrm{c} \mathrm{s}^{-1}$ and for almost three orders of magnitude, by a power-law with a slope $\alpha=-1.58 \pm 0.02$. The turn-over observed in the lower end of the frequency distribution may be attributed to detector sensitivity (missing the smaller events in the background noise). Figure 1a shows the frequency distribution of the flare total duration for the same timespan and the distribution can be represented by two powerlaws or a power-law with an exponential roll-over. The database was divided into five subgroups as function of their total duration $(D)$ : $6.5 \mathrm{~s}<D<200 \mathrm{~s}, 200 \mathrm{~s}<D<400 \mathrm{~s}$, $400 \mathrm{~s}<D<700 \mathrm{~s}, 700 \mathrm{~s}<D<1000 \mathrm{~s}, D>1000 \mathrm{~s}$. Frequency distributions were performed on the five subgroups and the two extremes ( $D<200 \mathrm{~s}$ and $D>1000$ s) are illustrated in Fig. 1d. All five frequency distributions can be represented by power-laws above a turn-over, but it is interesting to note that the slope of the power-law systematically varies with the range of the durations of the events (Table 1). This is due to the fact that the two parameters (peak count rate and duration) are positively correlated as can be seen in Fig. 1c. Georgoulis et al. (2001) were later able to reproduce efficiently most of the statistical properties found in this WATCH solar flare sub-grouping study by using cellular automata SOC models.

From a scale-invariant model like the avalanche model one would expect that the individual pulses that make up an avalanche would also show power-law behavior and that the slope values would be similar. The count rate frequency distribution of more than 5000 hard X-ray structures (less than $1 \mathrm{~s})$ detected in more than 600 solar flares was found to be well-represented by a power-law with a slope of -1.46 to -1.82 (Aschwanden et al., 1995, 1998). This is identical to what has been found for the global frequency distribution behavior of solar flare X-rays (e.g. Crosby et al., 1993) and thus supports the avalanche model. 


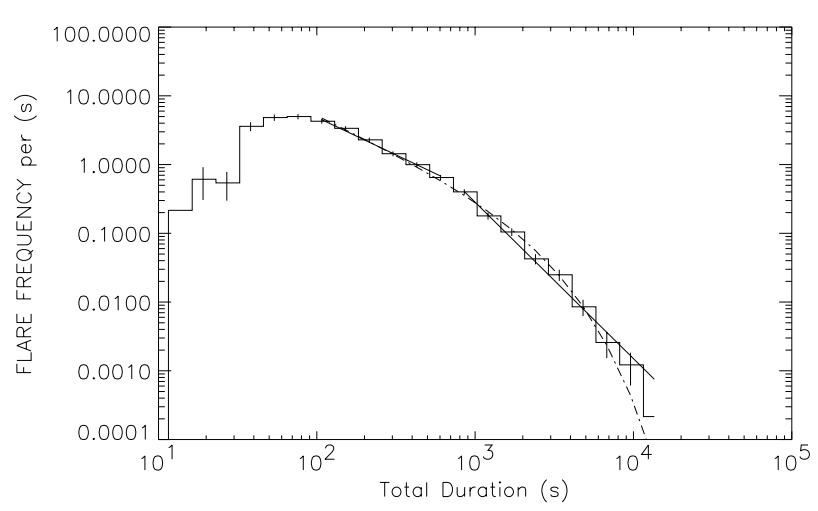

Fig. 1a. The frequency distribution of the WATCH total duration for the total observing period, that can either be represented by one single power-law with an exponential roll-over or by two powerlaws. From Crosby et al. (1998).

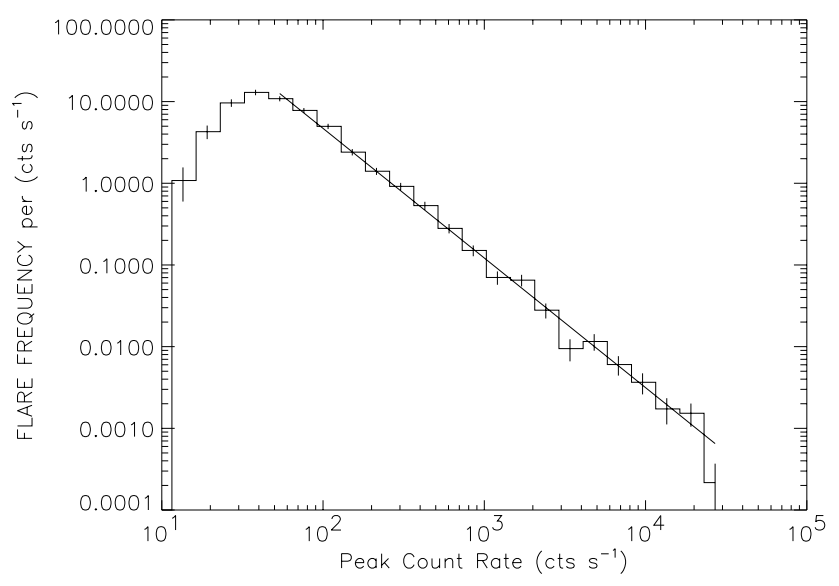

Fig. 1b. The frequency distribution of the WATCH flare peak count rate for the total observing period, that is well-represented by a power-law above the turn-over at $100 \mathrm{cs}^{-1}$ with a slope $-1.59 \pm 0.02$. From Crosby et al. (1998).

Often it is a measured parameter such as peak count rate or duration that is used in solar flare frequency distribution studies. For comparison reasons it is useful that modeled values such as the peak energy and total energy released in a solar flare be studied too. It was found that the frequency distribution of the total energy in electrons (ergs) computed for solar hard X-ray observations $(25-500 \mathrm{keV})$ is well-represented by a power-law with a slope of $-1.53 \pm 0.02$ (Crosby et al., 1993). For solar soft X-ray observations it was found that the frequency distribution of the energy contained in transient brightenings also follows a power-law with a similar slope [-1.5- -1.6] Shimizu (1995). This similarity in slope values suggested that independently on the form under which the released energy is converted (heating of plasma

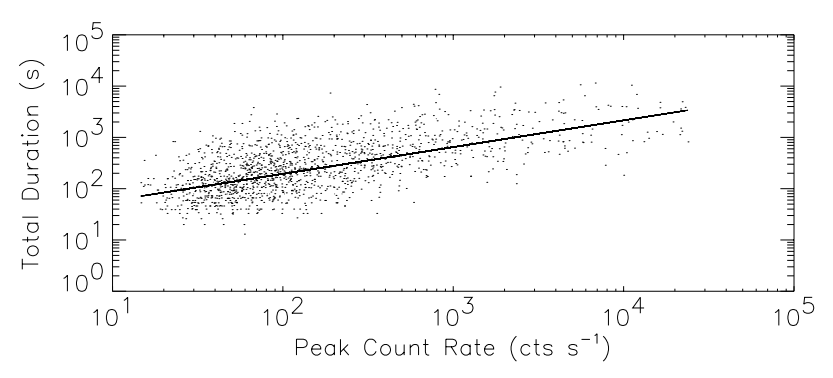

Fig. 1c. Scatter plot between the WATCH total duration with the peak count rate. From Crosby et al. (1998).

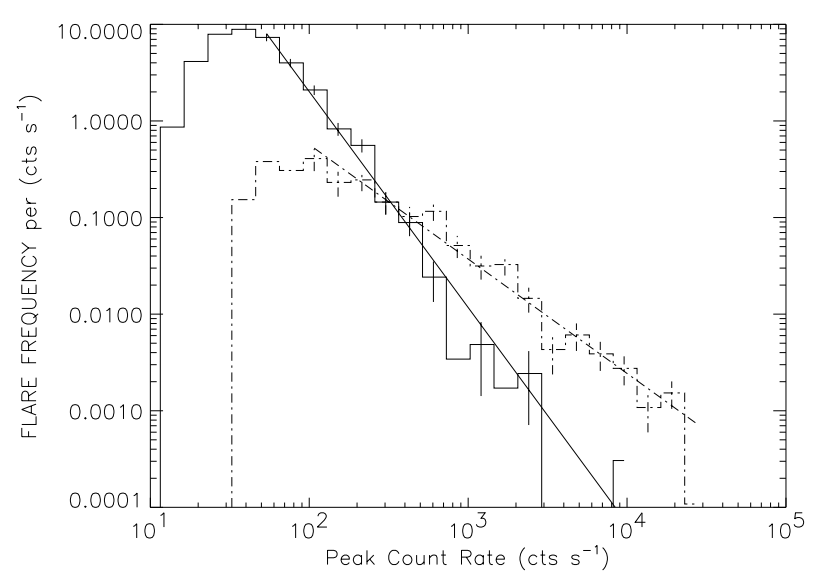

Fig. 1d. The frequency distribution of the WATCH peak count rate as function of duration interval. The steeper slope refers to events that have durations less than $200 \mathrm{~s}$ and the flatter slope to events that have durations greater than $800 \mathrm{~s}$. From Crosby et al. (1998).

or production of non-thermal particles), the analysis of frequency distributions of total energy contained in either nonthermal particles or hot plasmas gives a good identification on the energy release distribution itself. Furthermore by using previous performed frequency distributions of thermal energies from nanoflare statistics in the quiet Sun, Aschwanden (2004) found that the overall slope of the synthesized frequency nanoflare distribution is $-1.54 \pm 0.03$, similar to that of transient brightenings and X-ray flares (see Fig. 2).

Litvinenko (1996) uses a time-dependent continuity equation that that takes the dynamical evolution and mutual interaction of multiple magnetic reconnection current sites by coalescence into account and is able to quantify the frequency of flare energies, thus deriving a frequency distribution of flare energies with a power-law slope in the range of $E^{-1.5}$ and $\mathrm{E}^{-1.75}$. A physically based explanation for this similarity in energy frequency distribution slope values was given by Craig (2001) by showing that an $E^{-1.5}$ power-law is exactly the spectrum to be expected from distributed solar flare 
Table 1. Characteristics of the WATCH frequency distributions in peak count rate for sub-groups of events. $N$ fit: number of events in the fit. $N$ tot: total number of events in the distribution. Adapted from Crosby et al. (1998).

\begin{tabular}{llll}
\hline $\begin{array}{l}\text { Duration Interval } \\
\text { (s) }\end{array}$ & Slope & $N$ fit & $N$ tot \\
\hline$D>6.5$ & $-1.58 \pm 0.02$ & 1251 & 1537 \\
$6.5<D<200$ & $-2.17 \pm 0.07$ & 437 & 653 \\
$200<D<400$ & $-1.82 \pm 0.08$ & 236 & 309 \\
$400<D<700$ & $-1.46 \pm 0.06$ & 190 & 229 \\
$700<1000$ & $-1.34 \pm 0.03$ & 108 & 124 \\
$D>1000$ & $-1.15 \pm 0.05$ & 206 & 222 \\
\hline
\end{tabular}

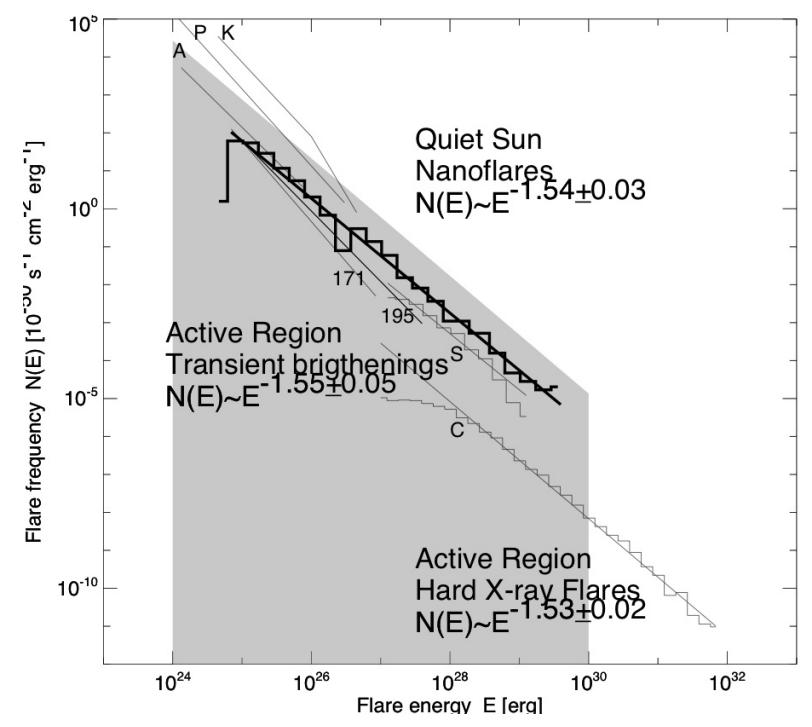

Fig. 2. Compilation of frequency distributions of thermal energies from nanoflare statistics in the quiet Sun, active region transient brightenings, and hard X-ray flares. The labels indicate the following studies: $\mathrm{K}=$ Krucker and Benz (1998), Benz and Krucker (2002), P = Parnell and Jupp (2000) (corrected for an error in the original paper), $\mathrm{A}=$ Aschwanden et al. (2000), Shimizu (1995), C = Crosby et al. (1993), and 171, $195=$ Aschwanden and Parnell (2002). From Aschwanden (2004).

reconnection events with 2-D current sheet geometry, using the known properties of analytic solutions for reconnection and a relatively conservative set of additional assumptions. Furthermore, Aschwanden and Parnell (2002) formulated a theory of frequency distributions and correlations of flarelike processes based on fractal geometry and the physical scaling laws known from the energy balance equation between heating, conductive, and radiative loss (RTV laws), using power-law approximations for the frequency distributions and scaling laws. Their theory reproduces a power-law slope value of -1.54 for the frequency distribution of thermal energies.

\subsection{Solar energetic particle events}

Sporadic solar energetic particle (SEP) events are the main concern for interplanetary space travel, especially at times of solar maximum. For mitigation purposes it is therefore essential to know the probability of a large SEP event occurring over a given time period. Like solar flares, SEP events also show power-law behavior over three to four decades when frequency distributions are performed on the data. Using data from some of the first space observatories (IMP-4 and IMP5) Van Hollebeke et al. (1975) performed frequency distributions on the number of proton events per unit intensity (maximum intensity at 40 MeV) from May 1967-December 1972 and found power-law behavior with a slope of $-1.15 \pm 0.05$. Around two decades later Cliver et al. (1991) obtained size distributions of peak fluxes of solar energetic proton (24 $32 \mathrm{MeV}$ ) and electron (3.6-18.5 MeV) events observed with particle detectors on IMP-8. They found that the peak differential fluxes of the proton events have a slope of $-1.30 \pm 0.07$ and for the electrons it was $-1.42 \pm 0.04$.

Gabriel and Feynman (1996) found power-law representations of time-integrated fluxes of solar energetic particle events using observations from IMP 1,2,3, OGO 1, and IMP $5,6,7$. Depending on the integral energy the slope values of the power-laws range are between -1.2 and -1.4 depending on the integral energy $(>10,30,60 \mathrm{MeV})$ over three to four orders of magnitude in fluence. A study by Miroshnichenko et al. (2001) found that a subset of sudden storm commencement associated events have a double power-law distribution with two exponents $(-1.00 \pm 0.04$ and $-1.53 \pm 0.03$ ), whereas the overall distribution has a slope value of $-1.37 \pm 0.05$. Other studies include Gerontidou et al. (2002) and references in the above mentioned SEP event studies.

The flatter value found for the SEP frequency distribution power-law slope compared to that found for solar flares could be a selection effect. The above studies were performed for proton energies greater than $10 \mathrm{MeV}$ meaning that the SEP events would have been associated with large solar flares and/ or coronal mass ejection shocks. Therefore one would automatically be covering more the region of high SEP flux values compared to low values, hence the flatter slope value. This is consistent with the flatter slope found for the WATCH peak count rate frequency distribution associated with longduration flares $(D>1000 \mathrm{~s})$; see Table 1 .

It is important to note that perhaps one has not yet measured the largest possible SEP event and that the largest SEP events used in empirical models originate only from the satellite era. McCracken et al. (2001a, b) analyzed a total of 125 large fluence SEPs identified from the nitrate deposition in ice core from Greenland for the period 1561-1950. These data have been augmented with ionospheric and satellite data for the period 1950-1994. There were five periods in the vicinity of $1610,1710,1790,1870$, and 1950 , 
when large $>30 \mathrm{MeV}$ proton events with fluence greater than $2 \times 10^{9} \mathrm{~cm}^{-2}$ were up to 8 times more frequent than in the era of satellite observation. The largest SEP in the nitrate record (associated with the Carrington white light flare event in 1859) had a $>30 \mathrm{MeV}$ proton fluence that was in the range $18-36 \times 10^{9} \mathrm{~cm}^{-2}$ (McCracken et al., 2001b). This is a factor 4-8 times greater than the value for the August 1972 solar particle event, frequently regarded as the "worst case" SEP event.

\section{Solar wind - magnetospheric signatures}

One of the fundamental problems of space physics is the characterization of global energy storage and release in the coupled solar wind-magnetosphere system. There exist two regimes of the solar wind (high speed and slow speed). Near solar minimum, activity is focused at low altitudes, highspeed solar wind prevails, and magnetic fields are dipolar, whereas near solar maximum, the solar winds are slower and more chaotic, with fluctuating magnetic fields. As the Sun rotates these various streams rotate as well (co-rotation) and produce a pattern in the solar wind much like that of a rotating lawn sprinkler.

As the solar wind flows past the Earth, it continuously energizes the terrestrial magnetosphere filling it with plasma and magnetic field energy. As the magnetosphere relaxes to lower-energy states, it releases the energy either back to the nightside solar wind, or deeper to the ionosphere. The magnetosphere can either dissipate the incoming energy directly, or store it and then discharge it. Usually it is a combination of both these processes. There are several ways for the energy transfer from the magnetosphere to the ionosphere to take place and one of them is through currents that connect the two regions. In the ionosphere it is the auroral electrojets which are the most intense ones and the most closely connected with the magnetospheric region where the energy is stored. At the same time the precipitating charged particles that form the currents give rise to the aurora.

The auroral zone geomagnetic energy is measured by an auroral electrojet index obtained from a number (usually greater than 10) of stations distributed in local time in the latitude region that is typical of the northern hemisphere auroral zone (Davis and Sugiura, 1966). For each of the stations the north-south magnetic perturbation $\mathrm{H}$ is recorded as a function of universal time. A superposition of these data from all the stations enables a lower bound or maximum negative excursion of the H component to be determined "the AL index". Similarly, an upper bound or maximum positive excursion in $\mathrm{H}$ is determined "the AU index". The difference between these two geomagnetic indices, $\mathrm{AU}-\mathrm{AL}$, is called the $\mathrm{AE}$ index (for more information see Mayaud, 1980).

Recent statistical studies of complexity in space plasmas have resulted in the search for SOC in the near-Earth space environment. Examples include the auroral electrojet
Table 2. Power-law slope values for the electron outer radiation belt count rate data (middle column) and the power-law slope value for the solar wind velocity (right-side column). Adapted from Crosby et al. (2005).

\begin{tabular}{lll}
\hline Year & $\begin{array}{l}\text { Electron Count Rate } \\
\text { slope }\end{array}$ & $\begin{array}{l}\text { Solar Wind Velocity } \\
\text { slope }\end{array}$ \\
\hline 1995 & $-1.476 \pm 0.007$ & $-5.54 \pm 0.15$ \\
1996 & $-1.655 \pm 0.006$ & $-10.45 \pm 0.27$ \\
1997 & $-1.728 \pm 0.008$ & $-13.76 \pm 0.59$ \\
\hline
\end{tabular}

indices, UVI auroral imagery, and in-situ measurements in the solar wind, Earth's magnetotail and Earth's outer electron radiation belt.

\subsection{Solar wind and auroral electrojet indices}

The solar wind speed is one of the important parameters for space weather prediction. It is especially at times of high speed solar winds that interesting effects are observed in the magnetosphere and often the higher the speed the more important is the effect.

Burlaga and Lazarus (2000) found that the lognormal distribution is a good model for the solar wind velocity data measured in 1996 to 1998 (less satisfactory for the data obtained during 1995 when corotating streams were present).

Frequency distributions of the solar wind velocity for the three years (1995, 1996 and 1997) were performed by Crosby et al. (2005) for all data and for two sub-groups of values: (1) corresponding to southward interplanetary magnetic field, (2) corresponding to northward interplanetary magnetic field. For each distribution the power-law fit of the solar wind velocity distribution for the interval $\left(500 \mathrm{~km} \mathrm{~s}^{-1}\right.$, $900 \mathrm{~km} \mathrm{~s}^{-1}$ ) was computed using the maximum likelihood method. Slopes for all three interplanetary magnetic field intervals lie within error bars and were found to be smallest in 1995 thereafter increasing systematically as a function of year (for all data see Table 2). Crosby et al. (2005) do not suggest that a power-law distribution is a better representation of the statistical properties observed in the solar wind velocity data than a lognormal distribution. Instead they look for trends in the slopes and emphasized using these results, that any realistic fit would show tendencies in the statistical properties of the data during the solar cycle.

Freeman et al. (2000) showed that the solar wind $v B_{\mathrm{S}}$ ( $v=$ solar wind speed, $B_{\mathrm{S}}=$ rectified function of the northsouth component) and $\epsilon$ burst lifetime distributions are of power-law form with an exponential cut-off, consistent with the solar wind being an SOC system. Furthermore, they showed that the $\epsilon$ burst lifetime distribution is not significantly different to that of the $\mathrm{AU}$ and $|\mathrm{AL}|$ geomagnetic indices, indicating that this scale-free property of the $\mathrm{AE}$ indices could arise from the solar wind input and may not be an intrinsic property of the magnetospheric system. 
However, based on the analysis of auroral electrojet index data, Uritsky et al. (2001), found an absence of one-toone mappings between integrated input power and AE bursts. They found that the solar wind input has essentially different scaling features and does not control the dynamics of the $\mathrm{AE}$ index bursts for times shorter than $3.5 \mathrm{~h}$ indicating the internal magnetospheric origin of the revealed effects. For reasons such as these Watkins (2002) suggested that better indicators of SOC behavior than those derived from $\mathrm{AE}$ are needed in the magnetospheric case.

\subsection{Auroral images}

Some of the most significant observations of SOC behavior in the magnetosphere have been found to be the scalefree statistical distributions of nighttime auroral emission regions. Lui et al. (2000) investigated whether the dynamic magnetosphere is an avalanching system by performing frequency distributions on parameters of the global auroral distribution by using auroral images obtained by the UV imager (UVI) on the Polar spacecraft. They found that the internal relaxations of the magnetosphere statistically follow powerlaws that have the same index independent of the overall level of activity (quiet vs sub-storm).

By extending the analysis of Lui et al. (2000) from a static spatial analysis to a spatiotemporal analysis of active regions in the Polar UVI images (Uritsky et al., 2002) showed that the probability distributions of the time-evolving blobs over the lifetime, maximum area, integrated area, maximum power and integrated energy output obey distinct power-laws over a wide range of scales. Especially, the integrated energy output can be represented by a power-law with a slope -1.5 over five orders of magnitude. Ground-based all-sky camera observations of auroral emission also show power-law behavior (Kozelov et al. 2004) close to those observed by Polar UVI image data (Uritsky et al., 2002, 2003). A study based on POLAR UVI images, show that energy and duration probability distributions of particle precipitation events obey finite-size scaling relations indicative of a SOC dynamical state (Uritsky et al., 2006a).

Time-dependent fractal measures of auroral activity can be used as sensitive indicators of early stages of the development of high-latitude geomagnetic disturbances providing important auxiliary information of phases of the nonlinear magnetospgheric response to the solar wind driver (Uritsky et al., 2006b). They highlight that this type of result suggests that during the development of geomagnetic perturbations, the auroral activity undergoes a transition from the SOC state to the super-critical state that is formed at the end of the growth phase or during the early expansion phase. This multiscale reorganization provides new quantitative information on the preparedness of the magnetosphere for a large-scale unloading that can improve the accuracy of space weather forecasting.

\subsection{Outer electron radiation belt}

Understanding the dynamic behavior of Earth's electron radiation belts is important for the design of spacecraft crossing this region (once or numerous times) and of spacecraft situated in geostationary orbit.

Even though there are no individually defined avalanches in the outer electron radiation belt, as there for example are in the solar corona (e.g. solar flares), one can imagine that the outer radiation belt acts as one individual system one "flare". As mentioned in Sect. 3.1 the frequency distribution of individual pulses making up a solar flare show power-law behavior. This inspired Crosby et al. (2005) to apply this technique on the outer electron radiation belt, the analogy being that the pulses that make up the outer radiation belt are identical with the burst structures making up a flare "individual avalanche".

In this study it was shown that frequency distributions of outer electron radiation belt data, measured by the CID/STRV-1 experiment $(>750 \mathrm{keV})$, are well-represented by power-laws over two decades. Furthermore sub-grouping the radiation belt count rate data as a function of spatial location or temporal interval (e.g. L-shell, magnetic local time, solar cycle) shows systematic trends in the value of the slope of the power-laws. Results suggest that the entire outer radiation belt appears to be affected as the sum of its individual parts. Furthermore trends observed in the data were similar to trends observed in the solar wind velocity distributions (see Table 2) linking the solar wind and the outer electron belt in agreement with earlier studies which used different techniques (e.g. Blake et al., 1997, Iles et al., 2002 and references therein).

There are various theories regarding electron radiation belt enhancements (e.g. acceleration mechanisms, loss mechanisms, relation to the solar driver) and performing frequency distributions on the simulated data is one way to test the models. In the end it may prove to be a mixture of physical processes that must be accounted for to be able to reproduce the signatures observed in the data.

\section{Phenomena on earth}

Earthquakes, floods, storms, volcanic eruptions, landslides, and forest fires are the major natural catastrophes that occur on Earth. Like their counterparts in space they too come in all sizes and durations. Concerning loss of life, earthquakes must be ranked first, but landslides too can be catastrophic wiping out villages in mountainous regions. The difference in hazard arises mainly from the fact that the seismic waves released by an earthquake cause damage on a regional scale, while the impact of landslides is often limited to smaller areas. In the following sub-sections some of these hazards will be discussed in regard to their frequency distribution signatures. 


\subsection{Earthquakes}

Earthquakes exhibit considerable complexity in their organization both in space and time but have also strong regularities. Long before the concept of SOC was introduced, earthquakes were known as a source of scale-invariant behavior such as the Gutenberg-Richter (G-R) Law describing the statistical distribution of earthquake sizes and by the Omori Law describing the frequency of aftershocks. The short time temporal correlation between earthquakes is given by the Omori Law (Omori, 1895), which states that immediately after an earthquake, the frequency of a sequence of aftershocks decays with time, where aftershocks are described as correlated events that occur after a large seismic event.

Over fifty years ago Gutenberg and Richter (1954) established the well-known Gutenberg and Richter size-frequency relationship that gives the number of earthquakes of magnitude larger than $\mathrm{M}$ in a large given geographic area over a long time interval, $\mathrm{N}(\mathrm{M})$. The law states that the frequencysize relationship of earthquakes scales according to the same power-law for all seismic regions, regardless of geological history and tectonic settings. The G-R law follows a powerlaw: $N=10^{a-b M}$, where $N=$ number of events having a magnitude $>\mathrm{M}, a$ and $b$ are constants. The constant $b$ is typically equal to 1.0 in seismically active regions. There is some variation with $b$-values in the range 0.5 to 1.5 depending on the tectonic environment of the region The G-R magnitude frequency distribution for energy released in earthquakes is the most commonly cited example of a naturally occurring SOC phenomenon on Earth and can be understood as a consequence of the Earth's crust being in a SOC state.

More recently Okubo and Aki (1987) demonstrated that the fault systems and the spatial distribution of epicenters of earthquakes are fractal. This was followed by Christensen et al. (2002) who proposed and verified a unified scaling law that describes the probability of interoccurrence times between earthquakes for a cutoff magnitude and region size. The Law links together the G-R Law, the Omori Law and the fractal dimension of the fault. Specifically, the Omori Law is shown to be the short time limit of a general hierarchical phenomenon containing the statistics of both main shocks and aftershocks indicating that they are created by the same mechanism.

It is generally accepted by the scientific community that the frequency size distribution of small, medium and large earthquakes follows a power-law. However, many works have investigated possible variations of this law from one seismic region to another and as a function of magnitude and time. Especially, the size distribution of the very rare, extreme big earthquakes is much less well understood and two main deviations have been reported and discussed repeatedly in the literature: (1) A turn-over is expected from general energy considerations. (2) The slope of the power-law for the same tectonic type is a function of ridge zones. For more details on these deviations and references therein, see Pisarenko et al. (2004a) and Pisarenko and Sornette (2004b).

\subsection{Landslides, wildfires, volcanoes, snow avalanches, rock-falls, etc.}

Landslides are complex natural phenomena that constitute a serious natural hazard in many countries. The landslide event is commonly associated with a trigger, such as an earthquake (minutes after), a rapid snowmelt (hours to days), or an intense rainfall (days to weeks), and ranges in size from a single landslide to many thousands. Frequency area distributions of medium and large landslides in each "inventory" decays as an inverse power of the landslide area over 2-4 orders of magnitude of landslide area (e.g. Malamud et al., 2004; Malamud, 2004).

Malamud et al. (1998) considered four forest fire and wildfire datasets from the USA and Australia. Thus the datasets came from a variety of geographic regions with different vegetation types and climate. In each case, it was found that the noncululative number of fires per year plotted as a function of burned fire area correlates well with a power-law relationship. The noncumulative frequency-area distribution of forest fires in the province of Ontario, Canada are shown in Turcotte and Malamud (2004).

Volcanoes erupt in different ways and thus come in all shapes and sizes. Power-law distributions indicating SOC behavior in volcano eruptions are mentioned in the literature. For example this behavior was found in the eruptions, volcano-induced earthquakes, dikes, fissures, lava flows, and interflow periods of the Piton de la Fournaise volcano by Grasso and Bachélery (1995).

Birkeland and Landry (2002) found evidence of frequency-size power-laws in several groups of snow avalanche paths and state that their results are consistent with SOC. They emphasize that the practical implication of this work is that the frequency-size relationship for small and medium sized avalanches may be useful for quantifying the risk of large snow avalanches within a group of avalanche paths.

Rockfalls studies also show power-law behavior, for example Dussauge et al. (2003) analyzed three rockfall data sets on subvertical cliffs and found that for all three catalogs the rockfall volume distribution follows a power-law distribution for volumes ranging from 102 to $1010 \mathrm{~m}^{3}$.

\section{Frequency distributions, forecasts, risk assessment and mitigation}

The tail of a distribution refers to its most extreme values. In the Gaussian distribution these tails are exponentials, while a distribution with a heavy tail decays much more slowly. A heavy tail is an attribute of a phenomenon called scale invariance, which as presented in the previous sections appears 
to underlie many natural systems in the Sun-Earth scenario. These phenomena are all found to have a frequency size distribution that follows a power-law. Hence, power-laws in these systems reveal an underlying universality in nature. More importantly, observing power-law behavior suggests that large natural hazards are not as infrequent as we might have originally thought.

It is important to note that by creating a frequency size distribution and using it to assess risks, one is assuming that the events themselves are uncorrelated in time (e.g. they are Poissonian). Therefore the main question "Are complex systems predictable or not?" is highly debated in the literature. As (Sornette, 2002) states it so concisely: "The outstanding scientific question that needs to be addressed to guide prediction is how large-scale patterns of a catastrophic nature might evolve from a series of interactions on the smallest and increasingly larger scales, where the rules for the interactions are presumed identifiable and known", as well as "It is essential to realize that the long-term behavior of these complex systems is often controlled in large part by these rare catastrophic events". For example, if large events do in fact cluster together significantly then it is important to include this in a risk analysis.

At present no proven method is available for the short term prediction of earthquakes (minutes to months). Current approaches can be divided into two general classes: (1) Empirical observations of precursory changes (e.g. seismic activity, ground motions). (2) Statistical patterns of seismicity. However, it is possible to make probabilistic hazard assessments for earthquake risks. Holliday et al. (2005) discuss a new approach to earthquake forecasting based on a pattern informatics method which quantifies temporal variations in seismicity. The output, which is based on an association of small earthquakes with future large earthquakes, is a map of areas in a seismic region ("hotspots") where earthquakes are forecast to occur in a future 10-yr time span.

Xapsos et al. (2000) developed a model predicting cumulative solar proton event fluence distributions using the Maximum Entropy approach and results correspond well with the measured solar proton distributions. Koons (2001) applied extreme value analysis to the magnetic index $A p$, solar proton daily averaged flux values, as well as to the excess of $>2 \mathrm{MeV}$ electron flux values over a threshold value at geosynchronous orbit. Results show that the extreme values observed to date are not unusual in that they are well fit by extreme value models. They also show that larger values than observed to date can be expected for each of the parameters during any $100-y r$ period. A similar type of analysis could be performed on data originating from other natural hazards.

Exponential roll-overs that are sometimes observed in the upper end of some frequency distributions could simply be due to missing data (all sizes of events have not been recorded in the given observing period). Perhaps what this means is that one simply has not yet observed over a systematically long enough time to have the total statistics of the phenomenon being studied. In such a case the discussion of whether the tail of the distribution is heavy tailed or not becomes irrelevant.

As long as there is enough statistics frequency distributions provide information on the statistical properties of the variability that is present in the natural system being observed on any chosen time-scale (e.g. months, years, decades). Furthermore this type of study also gives the probability of exceeding a given threshold value over a given time; limiting the size of an event which is important for mitigation purposes. The average values can then be compared with models used in mitigation strategies (e.g. spacecraft design, warning facilities,...). Power-law distributions are increasingly being used by reinsurance companies and governments to assess the risks posed by natural hazards, as power-laws allow one to make conservative and realistic estimates of these risks.

\section{SOC and frequency distributions}

As mentioned in Sect. 2 not only SOC models display power-law behavior. For this reason the following three SOC (physics-free) criteria have been proposed (Aschwanden, 2011):

- Statistical Independence: events that occur in a SOC system are statistically independent and not causally connected in space or time. Waiting time distributions should be consistent with a stationary or non-stationary Poisson process, in order to guarantee statistical independency by means of probabilities.

- Nonlinear Coherent Growth: time evolution of a SOC event has an initial nonlinear growth phase after exceeding a critical threshold. The nonlinear growth of dissipated energy, or an observed signal that is approximately proportional to the energy dissipation rate, exhibits an exponential-like or multiplicative time profile for coherent processes.

- Random Duration of Rise Times: if a system is in a state of SOC, the rise time or duration of the coherent growth phase of an avalanche is unpredictable and thus exhibits a random duration. The randomness of rise times can be verified from their statistical distributions being consistent with binomial, Poissonian, or exponential functions.

One difficulty in applying the SOC concept is its broad definition in the literature. Chapman and Watkins (2001) define three definitions: (1) Original SOC mechanism suggested by Bak et al. (1987), (2) Forced SOC introduced by Chang (1992), and (3) Phenomenological definition based on observation of some or all of a set of possible diagnostics of SOC such as bursty time series, " $1 / f$ " power 
spectra and avalanche distributions SOC-like (Chapman and Watkins (2001). Demonstration of SOC in the magnetosphere requires one to define a set of observable properties which are the unique fingerprint of SOC as stated by Watkins et al. (2001). Using auroral images in EUV is a promising fingerprint and the beauty of SOC is its way of connecting everything (wide field of applications). SOC is just one mechanism for explaining scale invariance and therefore not only SOC behavior produces power-laws.

However, it can not be ignored that many natural systems in the Sun-Earth scenario have frequency size distributions that follow a power-law distribution. Since power-laws are the only statistical distributions that are completely scaleinvariant, they offer a unique way to explore the possibility of an underlying universality in nature. By studying how the global statistics of avalanches vary for different phenomena one can in parallel not help but to ask the question: Does there exist a common avalanche signature? This is still a very important unanswered question.

DeArcangelis et al. (2006) analyzed available experimental earthquake and solar flare catalogs and showed that the stochastic processes underlying these apparently different phenomena have universal properties. Namely both problems exhibit the same distributions of sizes, inter-occurrence times and the same temporal clustering. The observed universality suggests a common approach to the interpretation of both phenomena in terms of the same driving physical mechanism. It should be noted here that direct measured parameters such as count rate will be detector dependant and this can influence the frequency distribution of the measured parameter. For example Aschwanden, Schwartz and Alt (1995) attributed the difference in the frequency distribution of solar flare hard X-ray pulses at $50 \mathrm{keV}$ with those at $25 \mathrm{keV}$ probably as a result of increased contamination in the lower energy range by thermal hard X-ray emission. In general if you compare solar flare peak count rate frequency distributions in the literature there are variations (see tables in Aschwanden, 2004). Therefore, it is suggested in this review paper that a derived parameter such as energy is more appropriate when comparing phenomenon, as certain instrumental effects will indirectly have been taken care of.

It was found that the frequency distribution of energy released in solar flares, transient brightenings, nanoflares (Sect. 3.1) and ionospheric emissions "auroral blobs" (Sect. 4.2) are similar. In these cases the slope value of the power-law is approximately -1.5 . Furthermore earthquakes have been found to have a similar distribution. This review paper suggests two things: (1) Energy may be released in a similar "universal" way, perhaps in a manner that maximizes the energy efficiency of the system where the phenomenon occurs. (2) The approximate value of 1.5 is a transcendental number in nature.

SOC is an interesting concept as its signature is not only found in natural systems. For example, Carreras et al. (2004) found that the blackout size time series in electrical power
Table 3. Analogy between a power system and a sandpile. Adapted from Carreras et al. (2004).

\begin{tabular}{lll}
\hline & Power System & Sand Pile \\
\hline System state & Loading pattern & Gradient Profile \\
Driving Force & Customer Load & Addition of Sand \\
Relaxing Event & Response to Blackout & Gravity \\
Event & Limit Flow or Trip & Sand Topples \\
\hline
\end{tabular}

systems seem indistinguishable from the sandpile avalanche size time series. This similarity suggests that SOC-like dynamics may play an important role in the global complex dynamics of power systems. Thus large blackouts are much more frequent than might be expected. In particular, the application of traditional risk evaluation methods can underestimate the risk of large blackouts. The analogy between a power system and a sand pile is presented in Table 3. One can only wonder where else similar tendencies of SOC behavior will be found in the future.

\section{Conclusions}

A simple approach, a powerful tool, frequency distributions provide us with very useful information for the understanding of the complex behavior found in dynamical systems. Modelling a given natural hazard as a complex system in a self-organized critical state provides a good context to understand the frequency distributions of the parameters describing the phenomenon and is one way of looking at it. It may turn out to be another theoretical model than SOC that can account for the observed power-law behavior (e.g. inverse cascade model) or it may be a mixture of many proccesses occuring simultaneously. However, the observations themselves provides one with useful information when performing frequency distribution on the data. The main conclusions of this review paper are as follows:

\section{Observational statistics}

- Frequency distributions performed on datasets describing natural dynamical phenomena (e.g. solar flares, earthquakes) exhibit power-law behavior.

- Subdividing the measured data as a function of a parameter and performing frequencing distributions on the sub-sets shows trends in the data revealing that the parameters are positively correlated.

Measurement problems and biases

- Turn-overs in the lower end of the frequency distribution for various phenomena may be attributed to detector sensitivity (missing the small events in the background noise). 
- Exponential turn-overs in the upper end of the frequency distribution for various phenomena may be due to either the length of the dataset (missing of long-term statistics) or that large events differ from their smaller counter-parts.

- Measured parameters are detector dependent and may give bias in the slopes of the frequency distributions for comparison purposes.

\section{Numerical SOC observations}

- Various concepts/models exist that produce powerlaw behavior such as self-organized criticality (SOC).

- SOC models are able to reproduce the results found when performing frequency distributions on measured data.

Statistical commonalities in SOC statistics

- Power-law behavior is found to be a universal characteristic defining natural dynamic phenomena (e.g. solar flares, earthquakes).

- Most distributions performed on observational data can be represented by power-laws having a slope in the range of $-1.4, \ldots,-2.4$.

- Frequency distributions of the energy released in solar flares, transient brightenings, nanoflares, ionospheric emissions and earthquakes are found to be similar (slope value of the power-law is approximate -1.5).

- This paper suggests that the energy parameter should be used for comparison purposes as it is detector independent.

Interpretations of physical processes

- Power-law frequency distributions result from nonlinear or coherent processes, have no charcteristic spatial scale and are the hallmark of nonlinear dissipative systems.

- Power-law frequency distributions of the energy released in some natural phenomena are found to be similar (slope value of the power-law is approximate -1.5$)$. This paper suggests:

- Energy is released in some type of universal way, perhaps in a manner that maximizes the energy efficiency of the system where the phenomenon occurs.

- The approximate value of 1.5 is a transcendental number in nature.
Mitigation and risk analysis

- Results from frequency distributions provide limits to the maximum strength of a phenomenon, vital for mitigation studies - probability of extreme events occurring (limit to the size of an event over a given time period).

- Implementing frequency distributions into the engineering approach "empirical models" is useful for design studies as well as probabilistic hazard assessment.

Acknowledgements. The author wishes to thank the Guest Editors of this Special Issue for inviting her to give a review talk at the associated workshop "Turbulence and Multifractals in Geophysics and Space". She also would like to acknowledge the referees of this review paper who took the time to provide useful feed-back thereby improving the objective of the paper. Finally, she would like to thank the editorial staff of the NPG journal.

Edited by: J. De Keyser

Reviewed by: M. Aschwanden and another anonymous referee

\section{References}

Aschwanden, M. J.: Physics of the Solar Corona, Springer Praxis Books Subseries: Geophysical Sciences, XIX, 858 pp., Hardcover ISBN:3-540-22321-5, 2004.

Aschwanden, M. J.: Self-Organized Criticality in Astrophysics, Springer Praxis Books, Subseries: Geophysical Sciences, XIX, 858 pp., Hardcover ISBN: ISBN: 978-3-642-15000-5, 2011.

Aschwanden, M. J. and Parnell, C. E.: Nanoflare Statistics from First Principles: Fractal Geometry and Temperature Synthesis, Astrophys. J., 572, 2, 1048-1071, 2002.

Aschwanden, M. J., Schwartz, R. A., and Alt, D. M.: Electron Time-of-Flight Differences in Solar Flares, Astrophys. J., 447, 923-935, 1995.

Aschwanden, M. J., Dennis, B. R., and Benz, A. O: Logistic Avalanche Processes, Elementary Time Structures, and Frequency Distributions in Solar Flares, Astrophys. J., 497, 972 993, 1998.

Aschwanden, M. J., Tarbell, T., Nightingale, R. W., Schrijver, C. J., Title, A., Kankelborg, C. C., Martens, P., and Warren, H. P: Time Variability of the "Quiet Sun Observed with TRACE. II. Physical Parameters, Temperature Evolution, and Energetics of ExtremeUltraviolet Nanoflares, Astrophys. J., 535, 2, 1047-1065, 2000.

Bai, T.: Variability of the occurrence frequency of solar flares as a function of peak hard X-ray rate, The Astrophysical Journal, Part 1 (ISSN 0004-637X), 404, 2, 805-809, 1993.

Bak, P.: How Nature Works, Springer-Verlag New York Inc., USA, 1996.

Bak, P., Tang, C., and Wiesenfeld, K.: Self-organized criticality An explanation of 1/f noise, Phys. Rev. Lett., 59, 381-384, 1987.

Bak, P. and Chen K.: Self-organized criticality, Scientific American, 264, 46-53, 1991.

Bak, P., Tang, C., and Wiesenfeld, K.: Self-organized criticality, Phys. Rev. A, 38/1, 364-374, 1988. 
Benz, A. and Krucker, A.: Energy Distribution of Microevents in the Quiet Solar Corona, Astrophys. J., 568, 1, 413-421, 2002.

Biesecker, D. A.: On the Occurrence of Solar Flares Observed with the Burst and Transient Source Experiment, $\mathrm{PhD}$ thesis, University of New Hampshire, 1994.

Biesecker, D. A., Ryan, J. M., and Fishman, G. J: Observations of Small Solar Flares with BATSE, in High-Energy Solar Phenomena - a New Era of Spacecraft Measurements, Proceedings of the Workshop Held in Waterville Valley, New Hampshire, March 1993, American Institute of Physics: New York, edited by: Ryan, J. and Vestrand, W. T., AIP Conference Proceedings, 294, 183 pp., 1994.

Birkeland, K. W and Landry, C. C.: Power-laws and snow avalanches, Geophys. Res. Lett., 29, 11, 2-4, 2002.

Blake, J. B., Baker, D. N., Turner, N., Ogilvie, K. W., and Lepping, R. P.:, Correlation of Changes in the Outer-Zone RelativisticElectron Population with Upstream Solar Wind and Magnetic Field Measurements, Geophys. Res. Lett., 24, 8, 927-930, 1997.

Bromund, K. R., McTiernan, J. M., and Kane, S. R.: Statistical studies of ISEE 3/ICE observations of impulsive hard X-ray solar flares, Astron. Astrophys., 455, 733-745, 1995.

Burlaga, L. F. and Lazarus, A. J.: Lognormal distributions and spectra of solar wind plasma fluctuations: Wind 1995-1998, J. Geophys. Res., 105, A2, 2357-2364, 2000.

Carlson, J. M. and Langer, J. S.: Properties of Earthquakes Generated by Fault Dynamics, Phys. Rev. Lett., 62, 2632-2635, 1989.

Carlson, J. M., Langer, J. S., and Shaw, B. E.: Dynamics of earthquake faults, Rev. Modern Phys., 66, 2, 657-670, 1994.

Carreras, B. A., Newman, D. E., Dobson, I., and Poole, A. B.: Evidence for Self-Organized Criticality in a Time Series of Electric Power System Blackouts, IEEE, 51, 9, 1733-1740, 2004.

Chang, T.: Low-Dimensional Behaviour and Symmetry Breaking of Stochastic Systems near Criticality - Can these Effects be Observed in Space and in the Laboratory, IEEE Trans. Plasma Sci., 20, 691, 1992.

Chang, T. S.: Self-organized criticality, multi-fractal spectra, sporadic localized reconnections and intermittent turbulence in the magnetotail, Phys. Plasmas, 6/11, 4137-4145, 1999.

Chapman, S. and Watkins, N.: Avalanching and Self-Organized Criticality, a Paradigm for Geomagnetic Activity, Space Sci. Rev., 95, 293-307, 2001.

Charbonneau, P., McIntosh, S.W., Liu, H.-L., and Bogdan, T. J.: Avalanche Models for Solar Flares, Solar Phys., 203, 2, 321353, 2001.

Christensen, K., Danon, L., Scanlon, T., and Bak, P.: Unified scaling law for earthquakes, PNAS, 99, 1, 2509-2513, 2002.

Cliver, E. W., Reames, D., Kahler, S., and Cane, H.: Size distributions of Solar Energetic Particle Events, in International Cosmic Ray Conference, 22nd, Dublin, Republic of Ireland, August 1123, 1991, Laboratory for High Energy Astrophysics Contributions (A92-36806 15-93). Greenbelt, MD, National Aeronautics and Space Administration, 2-1 to 2-4, 1991.

Cliver, E. W., Crosby, N. B., and Dennis, B. R.: Are Solar GammaRay Line Flares Different from other Large Flares?, Astrophys. J., 426, 767-773, 1994.

Craig, I. J. D.: A Reconnection Model for the Distribution of Flare Energies, Solar Phys., 202, 109-115, 2001.

Crosby, N.: Contribution à létude des Phénomènes Éruptifs du Soleil en Rayons X à partir des Observations de lÉxpérience
WATCH sur le Satellite GRANAT, Ph.D. thesis, Paris VII University, France, 1996.

Crosby, N. B., Aschwanden, M. J., and Dennis, B. R.: Frequency Distributions and Correlations of Solar X-Ray Flare Parameters, Solar Phys., 143, 275-299, 1993.

Crosby, N., Vilmer, N., Lund, N., and Sunyaev, R.: Deka-keV XRay Observations of Solar Bursts with WATCH/GRANAT: frequency distributions of burst parameters, Astronom. Astrophys., 334, 299-313, 1998.

Crosby, N. B., Meredith, N. P., Coates, A. J., and Iles, R. H. A.: Modelling the outer radiation belt as a complex system in a selforganised critical state, Nonlin. Processes Geophys., 12, 9931001, doi:10.5194/npg-12-993-2005, 2005.

Datlowe, D. W., Eclan, M. J., and Hudson, H. S.: OSO-7 Observations of Solar X-Rays in the Energy Range 10-100 keV, Solar Phys., 39, 155-174, 1974.

Davis, T. N. and Sugiura, M.: Auroral Electrojet Activity Index AE and its Universal Time Variations, J. Geophys. Res., 71, 785$801,1966$.

De Arcangelis, L., Godano, C., Lippiello, E., and Nicodemi, M.: Universality in Solar Flare and Earthquake Occurrence, Phys. Rev. Lett., 96, 5, 2006.

Dennis, B. R.: Solar Hard X-Ray Bursts, Solar Phys., 100, 465490, 1985.

Drossel, B. and Schwabl, F.: Self-organized critical forest-fire model, Phys. Rev. Lett., 69, 11, 1629-1632, 1992a.

Drossel, B. and Schwabl, F.: Self-organized criticality in a forestfire model, Physica A, 191, 1-4, 47-50, 1992b.

Dussauge, C., Grasso, J.-R., and Helmstetter, A.: Statistical analysis of rockfall volume distributions: Implications for rockfall dynamics, J. Geophys. Res., 108, 2286, doi:10.1029/2001JB000650, 2003.

Forrest, D. J.: Solar Gamma-Ray lines, in: Positron-Electron Pairs in Astrophysics, edited by: Burns, M. L., Harding, A. K., and Ramaty, R., A85-31276 13-90, AIP, NY, 3-4, 1983.

Freeman, M. P., Watkins, N. W., and Riley, D. J.: Evidence for a solar wind origin of the power burst lifetime distribution of the AE indices, Geophys. Res. Lett., 27, 8, 1087-1090, 2000.

Gabriel, S. B. and Feynman, J.: Power-law Distribution for Solar Energetic Proton Events, Solar Phys., 165, 337-346, 1996.

Galsgaard, K.: Investigations of numerical avalanches in a 3D vector field, Astron. Astrophys., 315, 312-318, 1996.

Geller, R. J., Jackson, D. D., Kagan, Y. Y., and Mulargia, F.: Earthquakes cannot be predicted, Science, 275, 1616-1617, 1997.

Georgoulis, M. and Vlahos, L.: Variability of the Occurrence Frequency of Solar Flares and the Statistical Flare, Astron. Astrophys., 336, 721-734, 1998.

Georgoulis, M., Vilmer, N., and Crosby, N.: A Comparison between statistical properties of solar X-ray flares and avalanche predictions in cellular automata statistical flare models, A\&A, 367, 326-338, 2001.

Gerontidou, M., Vassilaki, A., Mavromichalaki, H., and Kurt, V.: Frequency Distributions of Solar Proton Events, JASTP, 64, 5-6, 489-496, 2002.

Grasso, J. R. and Bachélery, P.: Hierarchial organization as a diagnostic approach to volcano mechanics: Validation on Piton de la Fournaise, Geophys. Res. Lett., 22, 21, 2897-2900, 1995.

Gutenberg, B. and Richter, C. F.: Seismicity of the Earth and associated Phenomena, Princeton University Press, 310 pp., 1954. 
Held, G. A., Solina, D. H., Solina, H., Keane, D. T., Haag, W. J., Horn, P. M., and Grinstein, G.: Experimental Study of Criticalmass Fluctuations in an Evolving Sandpile, Phys. Rev. Lett., 65, 9, 1120-1123, 1990.

Hergarten, S.: Self-Organized Criticality in Earth Systems, Springer, Germany, 2002.

Holliday, J. R., Nanjo, K. Z., Tiampo, K. F., Rundle, J. B., and Turcotte, D. L.: Earthquake forecasting and its verification, Nonlin. Processes Geophys., 12, 965-977, doi:10.5194/npg-12-9652005, 2005.

Horton, W. and Doxas, I.: A low-dimensional energy-conserving state space model for substorm dynamics, J. Geophys. Res., 101, A12, 27223-27238, 1996.

Hudson, H. S.: Threshold Effect in Second-Stage Acceleration, Solar Phys., 57, 237-240, 1978.

Iles, R. H. A., Fazakerley, A. N., Johnstone, A. D., Meredith, N. P., and Bühler, P.: The relativistic electron response in the outer radiation belt during magnetic storms, Ann. Geophys., 20, 957965, doi:10.5194/angeo-20-957-2002, 2002.

Isliker, H., Anastasiadis, A., and Vlahos, L.: MHD consistent cellular automata (CA) models. II. Applications to solar flares, Astron. Astrophys., 377, 1068-1080, 2001.

Jensen, H. J.: Self-Organized Criticality: Emergent Complex Behavior in Physical and Biological Systems (Cambridge Lecture Notes in Physics), Cambridge University Press, UK, 1998.

Kahler, S. W.: The role of the big flare syndrome in correlations of solar energetic proton fluxes and associated microwave burst parameters, J. Geophys. Res., 87, 3439-3448, 1982.

Klimas, A. J., Uritsky, V. M., Vassiliadis, D., and Baker, D. N.: Reconnection and scale-free avalanching in a driven current-sheet model, J. Geophys. Res., 109, A02218, doi:10.1029/2003JA010036, 2004.

Koons, H. C.: Statistical Analysis of Extreme Values in Space Science, J. Geophys. Res., 106, A6, 10915-10921, 2001.

Kozelov, B. V, Uritsky, V. M., and Klimas, A. J.: Power Law Probability Distributions of Multiscale Auroral Dynamics from Ground-Based TV Observations, Geophys. Res. Lett., 31, L20804, doi:10.1029/2004GL020962, 2004.

Krucker, S. and Benz, A.: Energy Distribution of Heating Processes in the Quiet Solar Corona, Astrophys. J., 501, L213-L216, 1998.

Kucera, T. A., Dennis, B. R., Schwartz, R. A., and Shaw, D: Evidence for a Cutoff in the Frequency Distribution of Solar Flares from Small Active Regions, Astrophys. J., 475, 338-347, 1997.

Lee, T. T., Petrosian, V., and McTiernan, J. M.: The Distribution of Flare Parameters and Implications for Coronal Heating, Astrophys. J., 412, 401-409, 1993.

Lin, R. P., Schwartz, R. A., Kane, S. R. Pelling, R. M., and Hurley, K. C.: Solar Hard X-Ray Microflares, Astrophys. J., 283, 421425, 1984.

Litvinenko, Y. E.: A new model for the distribution of solar flares, Solar Phys., 167, 321-331, 1996.

Lu, E. T.: Constraints on Energy Storage and Release Models for Astrophysical Transients and Solar Flares, Astrophys. J., 447, 416, doi:10.1086/175885, 1995.

Lu, E. T. and Hamilton, R. J.: Avalanches and the Distribution of Solar Flares, Astrophys. J., Part 2 - Letters (ISSN 0004-637X), 380, L89-L92, 1991.

Lu, E. T., Hamilton, R. J., McTiernan, J. M., and Bromund, K. R.: Solar Flares and Avalanches in Driven Dissipative Systems,
Astrophys. J., Part 1 (ISSN 0004-637X), 412, 2, 841-852, 1993. Lui, A. T. Y, Chapman, S. C., Liou, K., Newell, P. T., Meng, C. I., Brittnacher, M., and Parks, G.: Is the dynamic magnetosphere an avalanching system?, Geophys. Res. Lett., 27, 7, 911-914, 2000.

Malamud, B. D.: Tails of Natural Hazards, Physics World, 17, 3135, 2004

Malamud, B. D., Morein, G., and Turcotte, D. L.: Forest Fires: An Example of Self-Organized Critical Behavior, Science, 281, 1840-1842, 1998.

Malamud, B. D., Turcotte, D. L., Guzetti, F., and Reichenbach, P.: Landslides, Earthquakes, and Erosion, Earth Planet. Sci. Lett., 229, 45-59, 2004.

Mayaud, P. N.: Derivation, Meaning and Use of Geomagnetic Indices, Geophys. Monograph, 22, Am. Geophys. Union, Washington, D.C., 1980.

McCracken, K. G., Dreschhoff, G. A. M., Zeller, E. J., Smart, D. F., and Shea, M. A.: Solar cosmic ray events for the period 15611994, 1, Identification in polar ice, 1561-1950, J. Geophys. Res., 106, A10, 21585-21598, 2001a.

McCracken, K. G., Dreschhoff, G. A. M., Smart, D. F., and Shea, M. A.: Solar cosmic ray events for the period 1561-1994, 2, The Gleissberg periodicity, J. Geophys. Res., 106, A10, 21599 21609, 2001b.

Miroshnichenko, L. I., Mendoza, B., and Pérez-Enriquez, R.: Size Distrtibutions of the $>10 \mathrm{MeV}$ Solar Proton Events, Solar Phys., 202, 1, 151-171, 2001.

Okubo, P. G. and Aki, K.: Fractal geometry in the San Andreas fault system, J. Geophys. Res., 92, B1, 345-356, 1987.

Omori, F.: On the aftershocks of earthquakes, J. College Sci. Imper. Univ. Tokyo 7, 111-200, 1895.

O'Neill, T.: Geographica: Tsunamis- Where Next?, National Geographic, 207, p. 4, 2005.

Oztürk, S.: Temporal and three dimensional spatial analysis of Earthquake activity between 1970 and 2010 along the North Anatolian fault zone, Turkey, Journal of Concrete and Applicable Mathematics, 9, 9-16, http://www.eudoxuspress.com/ images/TOC-VOL-9--2011--JCAAM.pdf, 2011.

Parker, E. N.: Nanoflares and the Solar X-Ray Corona, Astrophys. J., 330, 474-479, 1988.

Parnell, C. E. and Jupp, P. E.: Statistical Analysis of the Energy Distribution of Nanoflares in the Quiet Sun, Astrophys. J., 529, 1, 554-569, 2000.

Pearce, G., Rowe, A. K., and Yeung, J.: A statistical analysis of hard X-Ray solar flares, Astrophys. Space Sci., 208, 1, 99-111, 1993.

Pisarenko, V. F. and Sornette, D.: Statistical detection and characterization of a deviation from the Gutenberg-Richter distribution above magnitude 8, Pure Appl. Geophys., 161, 4, 839-864, 2004b.

Pisarenko, V., Sornette, D., and Rodkin, M.: Deviations of the distributions of seismic energies from the Gutenberg-Richter law, Comput. Seismol., 35, 138-159, 2004a.

Robbrecht, E., Berghmans, D., and Van der Linden, R. A. M.: Automated LASCO CME Catalog for Solar Cycle 23: Are CMEs Scale Invariant?, Astrophys. J., 691, 1222, doi:10.1088/0004637X/691/2/1222, 2009.

Rosner, R. and Vaiana, G. S.: Cosmic flare transients - Constraints upon models for energy storage and release derived from the event frequency distribution, The Astrophysical Journal, Part 1, 
222, 15 June 1978 .

Schwartz, R. A., Dennis, B. R., Fishman, G. J., Meegan, C. A., Wilson, R. B., and Paciesas, W. S.: BATSE flare observations in Solar Cycle 22, In its The Compton Observatory Science Workshop, 457-468 (SEE N92-21874 12-90), 1992.

Shimizu T., Energetics and Occurrence Rate of Active-Region Transient Brightenings and Implications for the Heating of the Active-Region Corona, Publ. of the Astronomical Society of Japan, 47, 251-263, 1995.

Sornette, D.: Predictability of Catastrophic Events: Material Rupture, Earthquakes, Turbulence, Financial Crashes, and Human Birth, PNAS, 99, 1, 2522-2529, 2002.

Turcotte, D. L. and Malamud, B. D.: Landslides, Forest Fires, and Earthquakes: Examples of Self-Organized critical Behavior, Physica A, 340, 580-589, 2004.

Uritsky, V. M., Klimas, A. J., and Vassiliadis, D.: Comparative study of dynamical critical scaling in the auroral electrojet index versus solar wind fluctuations, Geophys. Res. Lett., 28, 19, 3809-3812, doi:10.1029/2001GL013026, 2001.

Uritsky, V. M., Klimas, A. J., Vassiliadis, D., Chua, D., and Parks, G.: Scale-Free Statistics of Spatiotemporal Auroral Emissions as Depicted by POLAR UVI Images: Dynamic Magnetosphere is an Avalanching System, J. Geophys. Res. (Space Physics), 107, A12, doi:10.1029/2001JA000281, 2002.

Uritsky, V. M., Klimas, A. J., and Vassiliadis, D.: Evaluation of spreading critical exponents from the spatiotemporal evolution of emission regions in the nighttime aurora, Geophys. Res. Lett., 30, 15, doi:10.1029/2002GL016556, 2003.
Uritsky, V. M., Klimas, A. J., and Vassiliadis, D.: Critical finite-size scaling of energy and lifetime probability distributions of auroral emissions, Geophys. Res. Lett., 33, 8 , doi:10.1029/2005GL025330, 2006a.

Uritsky, V. M., Klimas, A. J., and Vassiliadis, D.: Analysis and prediction of High-latitude Geomagnetic Disturbances Based on a Self-Organized Criticality Framework, Adv. Space Res., 37, 539-546, doi:10.1016/j.asr.2004.12.059, 2006b.

Uritsky, V. M., Paczuski, M., Davila, J. M., and Jones, S.I.: Coexistence of Self-Organized Criticality and Intermittent Turbulence in the Solar Corona, Phys. Rev. Lett., 99, 2, 2007.

Van Hollebeke, M. A. I., Ma Sung, L. S., and McDonald, F. B.: The Variation of Solar Proton Energy Spectra and Size Distribution with Heliolongitude, Solar Phys., 41, 189-223, 1975.

Watkins, N. W.: Scaling in the space climatology of the auroral indices: is SOC the only possible description?, Nonlin. Processes Geophys., 9, 389-397, doi:10.5194/npg-9-389-2002, 2002.

Watkins, N. W., Freeman, M. P., Chapman, S. C., and Dendy, R. O.: Testing the SOC Hypothesis for the Magnetosphere, JASTP, 63, 1435-1445, 2001.

Wheatland, M. S.: Do solar flares exhibit an interval-size relationship?, Solar Phys., 191, 381-389, 2000.

Xapsos, M. A., Summers, G. P., Barth, J. L., Stassinopoulos, E. G., and Burke, E. A.: Probabillity Model for Cumulative Solar Proton Event Fluences, IEEE Trans. Nucl. Sci., 47, 486-490, 2000. 